\title{
Kenya: L'identification des IAR demeure hasardeuse: la prévention est primordiale
}

Frontiers in Reproductive Health

Follow this and additional works at: https://knowledgecommons.popcouncil.org/departments_sbsr-rh

Part of the International Public Health Commons, Public Health Education and Promotion Commons, and the Women's Health Commons How does access to this work benefit you? Let us know!

\section{Recommended Citation}

"Kenya: L'identification des IAR demeure hasardeuse: la prévention est primordiale," FRONTIERES Résumés de Recherche Opérationnelle. Dakar: Population Council, 2000. 


\section{Kenya L'identification des IAR demeure Infections de L'Appareil Reproductif \\ $N^{\circ} 9$ hasardeuse : la prévention est primordiale \\ Plus de la moitié des clientes en planification familiale et en soins prénatals à l'hôpital de Nakuru au Kenya, étaient atteintes d'une ou de plusieurs infections de l'appareil reproductif. Environ un tiers de ces infections étaient sexuellement transmissibles. A l'aide d'algorithmes de prise en charge syndromique basée sur les symptômes dont faisaient état les femmes, les prestataires n'ont réussi à classifier correctement que 5 à 16 pourcent des femmes qui plus tard seront testées positives par les laboratoires. Etant donné les limites de la prise en charge syndromique, les programmes doivent mettre l'accent sur la prévention des Infections Sexuellement Transmissibles (IST).}

\section{Contexte}

Depuis 1990, le Conseil municipal de

Nakuru a mis en ouvre un programme multiforme destiné à réduire l'incidence des Infections de l'Appareil Reproductif (IAR), singulièrement celles qui sont sexuellement transmissibles, au nombre desquelles le VIH/SIDA. Les agents des cinq cliniques de la municipalité utilisent les directives de la prise en charge syndromique basée sur les symptômes rapportés par les clientes et les signes cliniques pour identifier les clientes atteintes d'IAR.

En 1998 déjà, le Population Council avait mené une étude pour vérifier la fiabilité de la prise en charge syndromique et déterminer la meilleure manière d'intégrer la prise en charge des IAR dans les Consultations Prénatales (CPN) et de Planification Familiale (PF) existantes. Les sources d'information incluaient : (1) les résultats d'examens médicaux dont, un examen pelvien, et celui des signes cliniques chez 906 clientes en PF, et 815 clientes en CPN ; (2) les facteurs de risque d'IST pour les clientes et leurs partenaires ; (3) des tests de laboratoire portant sur cinq IAR ; et (4) des entretiens avec 18 infirmières et 195 clientes. Après une évaluation des services d'IAR existantes, 18 infirmières des cinq hôpitaux municipaux ont bénéficié d'un cours de recyclage de trois jours en prise en charge syndromique, avec notamment une formation à l'utilisation d'une liste de contrôle pour la prise en charge des clientes.

\section{Résultats}

- Cinquante pourcent des clientes en PF et 59 pourcent des clientes en CPN souffraient d'au moins une IAR, comme l'ont montré les tests de laboratoire. Une proportion relativement élevée de clientes - 14 pourcent des clientes en PF et 21 pourcent des clientes en CPN - étaient affectées par une ou plusieurs IST (chlamydiae, gonococcies, trichomonas).

- Les infections vaginales dues à la vaginite bactérienne, au trichomonas et à la candidose étaient les plus courantes (47 pourcent de clientes en PF et 56 pourcent en $\mathrm{CPN}$ ), comparées aux infections du col de l'utérus dues aux gonococcies et/ou aux chlamydiae (7,5 pourcent des clientes en $\mathrm{PF}$ et 9,4 pourcent de celles en $\mathrm{CPN}$ ).

- Les femmes chez qui une IAR a été décelée grâce au test de laboratoire étaient asymptomatiques en majorité et ne manifestaient aucun signe clinique. Seules 23 à 29 pourcent des clientes en CPN et en PF infectées ont fait état d'un ou de plusieurs symptômes d'IAR, et chez 37 à 43 pourcent des clientes infectées les prestataires ont détecté des signes cliniques au moment de l'examen. 


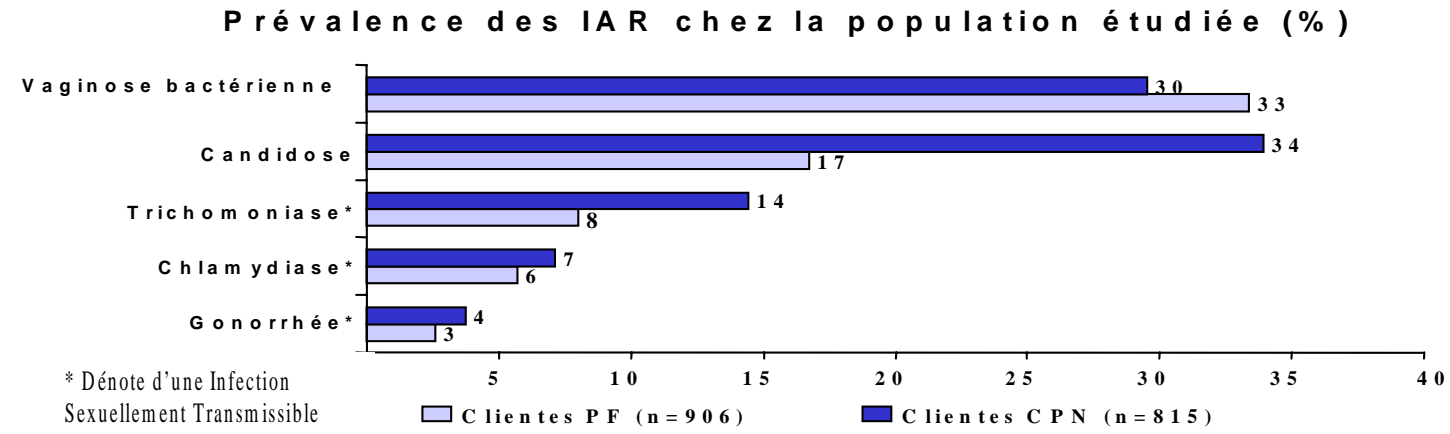

Avec les directives de la prise en charge syndromique, les prestataires n'ont été en mesure de classifier de manière correcte comme étant infectées qu'un faible pourcentage des femmes qui ont en réalité été diagnostiquées positives après un test de laboratoire IAR (5 pourcent des clientes en $\mathrm{PF}$ et 16 pourcent des clientes en $\mathrm{CPN}$ ).

- Les directives actuelles de prise en charge syndromique de femmes classifiées comme souffrant du syndrome de pertes vaginales sont plus fiables pour la prise en charge de femmes atteintes d'infections vaginales que pour celles qui souffrent d'infection du col de l'utérus. La majorité des femmes que les prestataires avaient classées comme souffrant du syndrome de pertes vaginales (61 pourcent des clientes en PF et

70 pourcent des clientes en CPN) souffraient en réalité d'infection vaginale, pendant que 11 pourcent seulement des clientes en PF et 8 pourcent des clientes en CPN, classifiées comme atteintes du syndrome de pertes vaginales, étaient affectées par une infection du col de l'utérus.

- La collecte d'informations sur l'évaluation des risques auprès des clientes n'a pas amélioré de manière significative la capacité des prestataires à identifier les femmes atteintes Implications pour les programmes

- Etant donné la modeste efficacité de la prise en charge syndromique pour les femmes qui présentent des pertes vaginales comme symptôme d'IST, les programmes doivent mettre l'accent sur le traitement, en améliorant les compétences des prestataires en matière de counseling, et en les encourageant à informer les clientes des symptômes des IST, ainsi que des mesures préventives, singulièrement la double protection.

- Si les programmes persistent à utiliser la prise en charge syndromique, alors les femmes considérées par les prestataires comme atteintes du syndrome de pertes vaginales devraient être d'abord traitées comme si elles souffraient d'une infection vaginale (vaginite bactérienne, candidose, et trichomonas), plutôt que si elles avaient une infection du col de l'utérus. Si les symptômes persistent, le traitement de l'infection du col de l'utérus peut être préconisé.

- Les algorithmes de prise en charge des pertes vaginales doivent être revus afin de mettre l'accent sur le traitement des vaginites bactériennes qui sont les IAR les plus courantes et qui ont été associées à un risque accru d'infection au VIH/SIDA et aux maladies inflammatoires pelviennes.

Mars 2000

d'infections du col de l'utérus

Solo, Julie ; Ndugga Maggwa ; James Kariba Wabur ; Bedan Kiare Kariuku ; et Gregory Maitha. 1999.

«Amélioration de la prise en charge des IST parmi les clientes en SMI/PF des hôpitaux municipal de Nakuru ». Pour de plus amples informations contacter : Population Council, P.O. Box 17643, Nairobi, Kenya. Tel. : 254-2-713-480; Fax : 254-2-713-479; E-mail: publications@popcouncil.or.ke

Ce projet a été exécuté avec le concours de l'AGENCE AMERICAINE POUR LE DEVELOPEMENT

INTERNATIONAL (USAID) aux termes du contrat numéro CCP-3030-C-00-3008-00 et des accords de coopération numéros CCP-3050-A-00-4013-00 et HRN-A-00-98-00012-00 